\title{
The added value of C-reactive protein to clinical signs and symptoms in patients with obstructive airway disease: results of a diagnostic study in primary care
}

\author{
Antonius Schneider*1, Geert-Jan Dinant ${ }^{2}$, Inko Maag ${ }^{1}$, Lutz Gantner ${ }^{1}$, \\ Joachim Franz Meyer ${ }^{3}$ and Joachim Szecsenyi ${ }^{1}$
}

\begin{abstract}
Address: ${ }^{1}$ University Medical Hospital Heidelberg, Dept. of General Practice and Health Services Research; Heidelberg, Germany, ${ }^{2}$ University Maastricht, Care and Public Health Research Institute, Dept. of General Practice, Maastricht, the Netherlands and ${ }^{3}$ University Medical Hospital Heidelberg, Dept. of Cardiology, Angiology and Pneumology, Heidelberg, Germany

Email: Antonius Schneider* - antonius.schneider@med.uni-heidelberg.de; Geert-Jan Dinant - geertjan.dinant@hag.unimaas.nl; Inko Maag - inkomaag@gmx.net; Lutz Gantner - lutzgantner@gmx.de; Joachim Franz Meyer - joachim.meyer@med.uni-heidelberg.de; Joachim Szecsenyi - joachim.szecsenyi@med.uni-heidelberg.de

* Corresponding author
\end{abstract}

Published: 02 May 2006

BMC Family Practice 2006, 7:28 doi:10.1186/147/-2296-7-28

This article is available from: http://www.biomedcentral.com/I47/-2296/7/28

(c) 2006 Schneider et al; licensee BioMed Central Ltd.

This is an Open Access article distributed under the terms of the Creative Commons Attribution License (http://creativecommons.org/licenses/by/2.0), which permits unrestricted use, distribution, and reproduction in any medium, provided the original work is properly cited.
Received: 12 December 2005

Accepted: 02 May 2006

\begin{abstract}
Background: To evaluate the diagnostic accuracy of clinical signs and symptoms, C-reactive protein (CRP) and spirometric parameters and determine their interrelation in patients suspected to have an obstructive airway disease (OAD) in primary care.

Methods: In a cross sectional diagnostic study, 60 adult patients coming to the general practitioner (GP) for the first-time with complaints suspicious for obstructive airway disease (OAD) underwent spirometry. Peak expiratory flow (PEF)-variability within two weeks was determined in patients with inconspicuous spirometry. Structured medical histories were documented and CRP was measured. The reference standard was the Tiffeneau ratio ( $\left.F E V_{1} / \mathrm{VC}\right)$ in spirometry and the PEFvariability. OAD was diagnosed when $\mathrm{FEV}, \mathrm{NC} \leq 70 \%$ or PEF-variability $>20 \%$.

Results: 37 (62\%) patients had OAD. The best cut-off value for CRP was found at $2 \mathrm{mg} / \mathrm{l}$ with a diagnostic odds ratio (OR) of 4.4 (95\% Cl 1.4-13.8). Self-reported wheezing was significantly related with OAD (OR 3.4; Cl I.I-I0.3), whereas coughing was inversely related $(\mathrm{OR} 0.2 ; \mathrm{Cl} 0.1-$ 0.7). The diagnostic OR of CRP increased when combined with dyspnea (OR 8.5; $95 \% \mathrm{Cl}$ I.7-42.3) or smoking history (OR 8.4; 95\% Cl I.5-48.9). CRP $(p=0.004), \mathrm{FEV}_{1}(p=0.00 \mathrm{I})$ and $\mathrm{FIV}_{1}(p=$ $0.023)$ were related with the severity of dyspnea. CRP increased with the number of cigarettes, expressed in pack years $(p=0.001)$.

Conclusion: The diagnostic accuracy of clinical signs and symptoms was low. The diagnostic accuracy of CRP improved in combination with dyspnea and smoking history. Due to their coherence with the severity of dyspnea and number of cigarettes respectively, CRP and spirometry might allow risk stratification of patients with OAD in primary care. Further studies need to be done to confirm these findings.
\end{abstract}




\section{Background}

The impact of obstructive airway diseases (OAD) is increasing, leading to high disability and mortality [1]. Consequently, optimal diagnostic performance is becoming an increasing challenge in primary care [2]. Medical history taking and physical examination deliver important information for diagnosing obstructive airway diseases $(\mathrm{OAD})$ in primary care, but their predictive values are weak $[3,4]$. Attempts were made to improve the diagnostic accuracy in this field, like the single measurement of peak expiratory flow (PEF) and the use of screening questionnaires $[5,6]$, but their sensitivities or specificities were low. Therefore, it would be helpful to find additional clinical markers to identify patients with risk for having an obstructive airway disease and to estimate their severity of the disease. One useful predictor for OAD could be Creactive protein (CRP), as it is related with irreversible airway obstruction [7] due to some kind of systemic inflammation [8].

However, the core of diagnostics is the spirometry and many efforts are done to implement its use in primary care $[9,10]$. Consequently, spirometers are increasingly available in general practice, and modern instruments are not only measuring $\mathrm{FEV}_{1}$ (forced expiratory volume in one second) and VC (vital capacity), but also inspiratory parameters. Inspiratory parameters could be of value for the assessment of the disease, as FIV (forced inspiratory volume in one second) was shown to be related with the severity of dyspnea $[11,12]$. The aim of the present study was to determine the diagnostic accuracy of clinical signs and symptoms and CRP for diagnosing airway obstruction in primary care. A secondary aim was to identify pre- dictors for the severity of the disease by analyzing the relation between clinical signs and symptoms, CRP and spirometric parameters.

\section{Methods \\ Design}

This cross sectional diagnostic study was performed between October 2003 and May 2004 in six general practices. The results from spirometry $\left(\mathrm{FEV}_{1} / \mathrm{VC}=\right.$ TiffeneauQuotient) and the variability of PEF-measurement within two weeks were used as a reference standard.

\section{Participants}

Sixty adult patients coming to the general practitioner for the first-time with complaints leading to the assumption of some kind of OAD were included consecutively. They presented symptoms like dyspnea, coughing, expectoration or self-reported wheezing. The patients have not been diagnosed earlier for OAD and they have not received any anti-obstructive medicine before. Other exclusion criteria related to well known side effects of inhaled sympathomimetics, namely untreated hyperthyreosis, unstable coronary artery disease or cardiac arrhythmia. Pregnancy also leads to exclusion. The study was approved by the Medical Ethics Committee of the University Heidelberg. Patients gave written informed consent.

\section{Measurements}

Medical history was taken with a structured interview similar to the asthma screening questionnaire of the European Community Respiratory Health Survey (ECRHS) [13]. This questionnaire was designed to assess the prevalence of asthma (= reversible OAD) in several European

Table I: Characteristics of the study population Values are numbers (proportion) or mean (SD); OAD = obstructive airway disease

\begin{tabular}{|c|c|c|c|c|}
\hline & $\begin{array}{l}\text { Overall } \\
\text { n (\%) }\end{array}$ & $\begin{array}{c}\text { No OAD or } \\
\text { restrictive pattern } \\
n(\%)\end{array}$ & $\begin{array}{c}\text { Reversible } \\
\text { OAD } \\
\text { n (\%) }\end{array}$ & $\begin{array}{c}\text { Not reversible } \\
\text { OAD } \\
\text { n (\%) }\end{array}$ \\
\hline Total study population & $60(100)$ & $23(38)$ & $27(45)$ & $10(17)$ \\
\hline Female & $40(67)$ & $15(65)$ & $19(70)$ & $6(60)$ \\
\hline Age [mean (SD)] & $46(18)$ & $42(18)$ & $46(17)$ & $54(18)$ \\
\hline Have you had wheezing in your chest (yes) & $39(65)$ & II (48) & $22(82)$ & $6(60)$ \\
\hline Do you sometimes have dyspnea (yes) & $35(58)$ & $12(52)$ & $16(59)$ & $7(70)$ \\
\hline Do you often cough (yes) & $26(43)$ & $15(65)$ & $9(33)$ & $2(20)$ \\
\hline Do you often have expectoration (yes) & $22(37)$ & $7(30)$ & $11(4 I)$ & $4(40)$ \\
\hline Wheezing when not having a cold (yes) & $25(42)$ & $7(30)$ & $15(56)$ & $3(30)$ \\
\hline Breathless when wheezing was present (yes) & $22(37)$ & $7(30)$ & II (4I) & $4(40)$ \\
\hline Have you been woken up with a feeling of tightness in your chest (yes) & $14(23)$ & $6(26)$ & $5(19)$ & $3(30)$ \\
\hline Have you been woken up by an attack of dyspnea (yes) & $13(22)$ & $6(26)$ & $7(26)$ & $0(0)$ \\
\hline Have you been woken up by an attack of coughing (yes) & $39(65)$ & $16(70)$ & $17(63)$ & $6(60)$ \\
\hline Do you have any nasal allergies (yes) & $25(42)$ & $10(43)$ & $12(44)$ & $3(30)$ \\
\hline Do you often have common cold (yes) & $22(37)$ & $8(35)$ & $8(30)$ & $6(60)$ \\
\hline Does it often take longer than 10 days to recover from a common cold (yes) & $29(48)$ & $10(43)$ & $12(44)$ & $7(70)$ \\
\hline Do you smoke/Did you smoke (yes) & $35(58)$ & $12(52)$ & $16(59)$ & $7(70)$ \\
\hline How much do you smoke [mean in pack year (SD)] & $13(15)$ & $13(10)$ & $9(10)$ & $21(26)$ \\
\hline
\end{tabular}


areas. The interview was expanded to symptoms of COPD (= irreversible OAD) (table 1). Patients were asked for the severity of their dyspnea, categorized as dyspnea at rest (severe); dyspnea while walking straight ahead (moderate); dyspnea while taking the stairs of two floors (mild) and no dyspnea. Patients were questioned about their smoking habits, allowing calculation of the number of pack years (= [years of smoking: 20$]$ × number of daily cigarettes). The physician performed the physical examination, always including auscultation of the patients' lungs. All findings were documented and checked for completeness.

Spirometry was performed in the general practices by two research assistants, who were initially trained at the pulmonary outpatient clinic of the University Hospital of Heidelberg. In case of an unacceptable flow-volume curve, the spirometric software gave an error message. The best of three consecutive spirometry recordings was used, according to the guidelines of the American Thoracic Society (ATS) [14]. The maximal inspiratory and expiratory flow volume curves were generated by forced deep inspiration and expiration with short intervening periods of tidal breathing. The forced inspiratory manoeuvres were always done after forced expiratory manoeuvres.

Every patient received a bronchodilation test with an additional performance of spirometry 20 minutes after inhaling Salbutamol through a spacer. An obstructive airway disease was diagnosed if $\mathrm{FEV}_{1} / \mathrm{VC} \leq 70 \%$. The obstruction was classified as not reversible on Salbutamol, if the bronchodilation response $\Delta \mathrm{FEV}_{1}$ was below $12 \%$ of the baseline and below $200 \mathrm{ml}$ [15]. Blood was taken after the spirometry manoeuvre for determining $\mathrm{CRP}$, which was assessed with an automated chemiluminometric assay kit.

Patients with no signs of airway obstruction after the results of the spirometry (i.e. $\mathrm{FEV}_{1} / \mathrm{VC}>70 \%$ ) received a PEF-meter (Vitalograph ${ }^{\circledast}$ ) in order to perform PEF-measurements twice daily for 14 days. The patients were trained in performing this maneuver and documented the results in a diary. If the variability ((highest value - lowest value)/highest value) within these two weeks was above $20 \%$, reversible airway obstruction (= asthma) was diagnosed according to the international asthma guidelines [15]. The final diagnosis (reference standard) was confirmed by the main investigator (A.S.) and the pulmonologist (J.M.), who were kept unaware of the measured inspiratory parameters and CRP.

\section{Data analysis}

As the sensitivities and specificities of clinical signs and symptoms and spirometric parameters are yet unknown for OAD diagnosed in primary care, a proper power calcu- lation could not be performed. The data were analyzed with SPSS 11.0 for Windows.

The CRP values were log-transformed and entered the ROC-analyses to assess the best cut-off point. Sensitivity, specificity, and predictive values were calculated from $2 \times$ 2 tables at the best cut-off-point. The best cut-off-point was chosen at the highest sum of positive and negative predictive value (PPV and NPV). CRP and clinical signs and symptoms were compared with the reference outcomes of spirometry and PEF-protocol. 95\% confidence intervals were calculated using Wilson's method [16]. Binary logistic regression was done to estimate the diagnostic odds ratios (OR) of CRP and clinical signs and symptoms (as independent variables) for the presence of OAD (as dependent variable). Positive likelihood ratios $(\mathrm{LR}+)$ were calculated to receive the ratio of abnormal findings in ill and healthy patients. Negative likelihood ratios (LR-) were calculated for the ratio of normal findings in ill and healthy patients. 95\% confidence intervals were derived from the log method [17].

In subgroup analyses, diagnostic values of CRP were calculated in combination with dyspnea, smoking history, coughing, expectoration, wheezing and abnormal finding in auscultation. In patients with the established diagnosis of OAD, the relationships between severity of dyspnea, CRP and spirometric parameters respectively were investigated using Spearman's correlation coefficient (for ordinal variables) and Pearson's correlation coefficient (for continuous variables).

\section{Results \\ Study population}

Two-thirds of the 60 patients were female; the average age was 46 years. 27 (45\%) had a reversible OAD, of which 8 were identified by spirometry and 19 through the PEFprotocol (figure 1).

Ten showed an airway obstruction irreversible after inhalation of Salbutamol; one patient had a restrictive pattern. Fourteen (23\%) patients with PEF-variability below $20 \%$ within two weeks did not show abnormal signs in spirometry. Eight (13\%) patients with inconspicuous spirometry did not bring back the PEF-protocol, assuring that they had no problems anymore. Thus, in total 37 (62\%) did have OAD, whereas $23(38 \%)$ patients were diagnosed as not having OAD.

The patients without OAD were four years younger than those with reversible OAD and the latter ones were eight years younger than those with not reversible OAD (table $1)$. The patients complained most frequently about wheezing (65\%) and dyspnea (58\%), coughing (43\%) and expectoration (37\%). Dyspnea and expectoration 


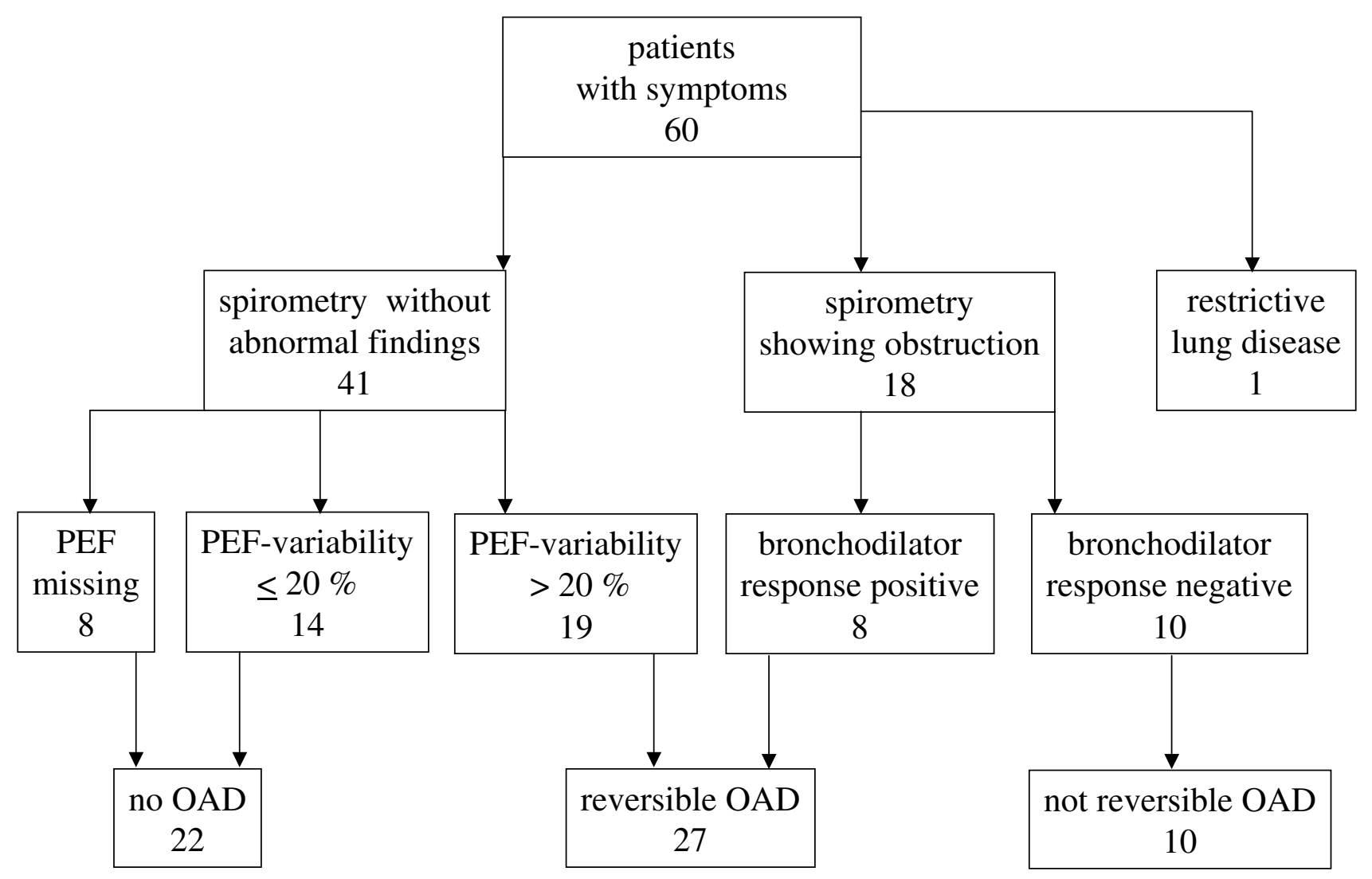

Figure I

Flow chart of inclusion and diagnostic work up (OAD = obstructive airway disease; PEF = peak expiratory flow).

were mainly associated with $\mathrm{OAD}$, whereas coughing was related with not having $\mathrm{OAD}$. Wheezing was more pronounced for those having OAD. Waking up by an attack of coughing seems not to distinguish healthy from ill patients, as there is a similar distribution in all patients. Patients with non-reversible OAD seem to have a common cold (60\%) more often, taking more than 10 days for recovering $(70 \%)$. These patients also had the highest tobacco use (21 pack years). Those with reversible OAD had the lowest use ( 9 pack years).

Diagnostic value of CRP and clinical signs and symptoms The best cut-off point for CRP was at $2 \mathrm{mg} / \mathrm{l}$, showing a diagnostic OR of 4.4 (table 2). The five symptoms with

Table 2: Sensitivities (sens), specificities (spec), positive and negative predictive values (PPV and NPV), odds ratios (OR) and likelihood ratios (LR+ and LR-) of clinical signs and symptoms and C-reactive protein $(C R P)$ for the presence of obstructive airway disease $(n=$ 60)

\begin{tabular}{|c|c|c|c|c|c|c|c|c|c|c|c|c|c|c|}
\hline Signs/Symptoms & Sens & $95 \% \mathrm{Cl}$ & Spec & $95 \% \mathrm{Cl}$ & PPV & $95 \% \mathrm{Cl}$ & NPV & $95 \% \mathrm{Cl}$ & OR & $95 \% \mathrm{Cl}$ & LR + & $95 \% \mathrm{Cl}$ & LR - & $95 \% \mathrm{Cl}$ \\
\hline CRP $>2 \mathrm{mg} / \mathrm{l}$ & & & & & & & & & & & & & & \\
\hline Dyspnea & 62.2 & $46.1-75.9$ & & & & & & & & & & & & \\
\hline Coughing & & & & & & & & & & & & & & \\
\hline & & $26.3-56.5$ & & & & & & & & & & & 0.9 & \\
\hline Wheezing & 75.7 & $59.9-86.6$ & 52.2 & $33.0-70.8$ & 71.8 & $56.2-83.5$ & & & 3.4 & $1.1-10.3$ & 1.6 & & 0.5 & \\
\hline Smoking history & 62.2 & $46.1-75.9$ & 52.2 & $29.2-67.0$ & 65.7 & $49.2-79.2$ & 44.0 & $26.7-62.9$ & 0.7 & $0.2-1.9$ & 1.2 & $0.8-1.9$ & 0.8 & $0.4-1.4$ \\
\hline Auscultation & 27.8 & $15.8-44.0$ & 87.0 & 67.9-95.5 & 76.9 & $49.7-91.8$ & 43.5 & $30.2-57.8$ & 2.6 & $0.6-10.6$ & 2.1 & $0.7-6.9$ & 0.8 & $0.6-1.1$ \\
\hline
\end{tabular}


Table 3: Relation between severity of dyspnea, CRP, FEVI and FIVI in patients with airway obstruction

\begin{tabular}{lcccccc}
\hline Severity & $\mathbf{n}$ & $\begin{array}{c}\text { CRP [mg/I] } \\
\text { mean (sd) }\end{array}$ & $\begin{array}{c}\text { FEV } \text { [I] } \\
\text { mean (sd) }\end{array}$ & $\begin{array}{c}\text { FEV , pred. [\%] } \\
\text { mean (sd) }\end{array}$ & $\begin{array}{c}\text { FIV, [I] } \\
\text { mean (sd) }\end{array}$ & $\begin{array}{c}\text { Smoking [py] } \\
\text { mean (sd) }\end{array}$ \\
\hline No dyspnea & 14 & $2.8(1.5)$ & $3.4(1.0)$ & $94.6(22.7)$ & $3.65(0.8)$ & $4.4(6.1)$ \\
Mild & 16 & $7.1(9.2)$ & $2.4(0.9)$ & $83.8(16.9)$ & $2.9(1.1)$ & $9.9(20.9)$ \\
Moderate & 2 & $6.7(1.1)$ & $1.8(0.4)$ & $69.0(21.8)$ & $2.6(0.1)$ & $5.0(7.1)$ \\
Severe & 5 & $11.6(13.1)$ & $2.1(1.1)$ & $82.6(16.0)$ & $2.5(1.2)$ & $12.7(8.2)$ \\
P & & $\mathbf{0 . 0 0 4}$ & $\mathbf{0 . 0 0 1}$ & 0.079 & $\mathbf{0 . 0 1 2}$ & 0.474
\end{tabular}

Py = pack year

the best test characteristics are listed in table 2. Only self reported wheezing was significantly associated with having OAD (OR 3.4), showing a sensitivity of $75.7 \%$ and specificity of $52.2 \%$. The sensitivity of "often coughing" was only $29.7 \%$ and the specificity was $34.8 \%$. Thus, coughing was negatively related with OAD (OR 0.2). Moreover, auscultation was not significantly associated with the diagnosis.

The diagnostic odds ratio of CRP increased when it was combined with the clinical symptom dyspnea (OR 8.5; $95 \%$ CI 1.7-42.3) or a positive smoking history (OR 8.4; 95\% CI 1.5-48.9) (not in table). However, confidence intervals were wide because of small sample sizes $(n=35$ in both subgroups). There was no significant improvement when CRP was combined with the other clinical signs and symptoms.

\section{Relation between severity of dyspnea, smoking history, CRP and spirometric parameters}

There was a significant association between CRP and the severity of dyspnea in patients with established diagnosis (correlation coefficient $t_{\text {Spearman }}\left[\mathrm{CO}_{\mathrm{sp}}\right]=0.46$ ) (table 3 ). There was also a significant relationship between severity of dyspnea and decrease of $\mathrm{FEV}_{1}\left(\mathrm{CO}_{\mathrm{sp}}=-0.52\right)$ and $\mathrm{FIV}_{1}$ $\left(\mathrm{CO}_{\mathrm{sp}}=-0.41\right)$. CRP increased with the number of pack years $($ correlation coefficient Pearson $=0.648)($ table 4$)$.

\section{Discussion}

This study demonstrates the difficulties in diagnosing obstructive airway diseases in general practice solely on the basis of clinical signs and symptoms. A combination of clinical signs and symptoms with CRP had the best diagnostic accuracy. CRP was related to smoking history; and it could be shown for the first time that CRP is related to the severity of dyspnea in patients with OAD. Also FEV and FIV $_{1}$ were associated with the severity of dyspnea.

Only self reported wheezing was positively associated with the presence of the disease, but the other parameters of medical history were not accurate for identifying patients suffering from OAD. These findings are consistent with previous studies [18-20]. Interestingly, coughing is negatively associated with the presence of OAD. This might contradict common clinical findings [3], but these are often derived from selected clinical settings. Coughing also had a negative association in one survey, pointing out that this symptom is often attributed to other illnesses than asthma [18]. The results of auscultation are similar to those from Strauss et al, who found a positive LR of 2.7 in his survey. Thus, auscultation could be a difficult marker when the patient is not symptomatic during the physical examination.

The relation between CRP and the severity of airway obstruction in COPD was illustrated before in a population based survey, which found an association between $\mathrm{FEV}_{1}$ and CRP [8]. CRP has attracted more attention over the last years, as multiple associations with cardiovascular diseases [21], COPD [7], osteoporosis [22] and even depression [23] were identified. It is speculated that some of these diseases are caused by a low grade inflammation reflected by a small elevation of inflammatory markers [8], which might indicate a higher prevalence of systemic complications. Our findings also confirm that there is a chronic inflammatory process on a low level, since a no more than slightly elevated CRP ( $>2 \mathrm{mg} / \mathrm{l}$ ) has a significant diagnostic OR for OAD. This cut-off-point is in line

Table 4: Relation between smoking history, CRP, FEVI and FIVI in patients with airway obstruction

\begin{tabular}{|c|c|c|c|c|c|}
\hline Smoking [pack year] & $\mathbf{n}$ & $\begin{array}{c}\mathbf{C R P}[\mathbf{m g} / \mathbf{l}] \\
\text { mean (sd) }\end{array}$ & $\begin{array}{l}\mathbf{F E V},[\mathbf{l}] \\
\text { mean (sd) }\end{array}$ & $\begin{array}{c}\text { FEV } \text {,pred. [\%] } \\
\text { mean (sd) }\end{array}$ & $\begin{array}{c}\mathbf{F I V} \text {, [I] } \\
\text { mean (sd) }\end{array}$ \\
\hline $0<\mathrm{py} \leq 5$ & 10 & $2.9(1.6)$ & $2.7(1.0)$ & $79.1(22.0)$ & $3.2(0.1)$ \\
\hline $5<p y \leq 10$ & 3 & $3.2(2.3)$ & $3.0(0.8)$ & $89.8(4.8)$ & $3.5(0.8)$ \\
\hline $10<$ py $\leq 20$ & 6 & $5.3(4.2)$ & $3.2(1.4)$ & $93.6(14.6)$ & $3.3(1.1)$ \\
\hline py $>20$ & 4 & $13.7(8.4)$ & $2.7(1.4)$ & $82.1(13.4)$ & $3.3(2.1)$ \\
\hline $\mathrm{p}$ & & 0.001 & 0.381 & 0.552 & 0.147 \\
\hline
\end{tabular}


with Sin et al. [8] who found their best threshold value for CRP at $2.2 \mathrm{mg} / \mathrm{l}$. Our finding of a positive association between CRP and the number of pack years is supported by Gan et al., who detected that active smoking increases CRP [24]. They suggested that smoking and reduced $\mathrm{FEV}_{1}$ have an additive effect on systemic inflammation. The association with the severity of dyspnea and smoking history might make CRP of interest for risk stratification of patients with OAD in primary care. The importance of this has been underlined by Huijnen et al., who identified dyspnea as a significant predictor for mortality [25]. Until now, the diagnostic value of CRP for primary care was mostly evaluated for acute inflammatory diseases like lower respiratory tract infection (LRTI) [26] and acute maxillary sinusitis [27]. In LRTI, CRP has a high diagnostic value in combination with clinical signs and symptoms [26]. The impact on practice management was demonstrated as the use of CRP lowered antibiotic prescribing for sinusitis [28]. In our study the diagnostic odds ratios of CRP improved in patients with dyspnea or smoking history. However, the confidence intervals were wide, which could mainly be due to the small sample size. And it must be noted additionally that it was not possible to differentiate between asthma and COPD. Therefore, further studies in larger populations of patients with COPD and asthma are necessary.

We were the first to demonstrate in primary care that dyspnea is correlated with $\mathrm{FIV}_{1}$. This relation was so far only demonstrated in a highly selected population with already known COPD $[11,12]$. Taube et al found, that the perception of dyspnea is more related to the inspiratory

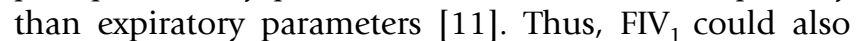
provide possibilities for risk stratification of patients in primary care as it is related with the severity of dyspnea. However, it must be noted for our study, that FEV shows $_{1}$ a higher correlation coefficient than FIV $_{1}$. Therefore the applicability and specificity of these parameters for primary care need to be evaluated in further studies.

There are some more limitations of our study. A reason for underestimation of the prevalence of OAD could be that eight patients did not bring back the PEF-meter with the protocol. However, they assured that they had no problems any more. One could argue that the performance of bronchoprovocation in a specialized centre would have been the best gold standard to evaluate the accuracy of spirometry in primary care and to distinguish between COPD and asthma. As this was not available in general practice, we tried to get as close as possible to the theoretical gold standard [29]. In a highly selected clinical population the measurement of PEF-variability within two weeks had a smaller sensitivity and specificity than bronchoprovocation [30]. However, the value of bronchoprovocation should not be over-estimated as a poor agreement between bronchial hyper-reactivity and clinical asthma was demonstrated in a review [31], and the correlation between the clinical diagnosis asthma and bronchoprovocation could be low [32]. Classical test characteristics derived from hospital studies are of limited value in primary care due to the lower incidence and smaller extent of the particular disease found there [33].

\section{Conclusion}

The diagnostic accuracy of clinical signs and symptoms was low as only self-reported wheezing had a significant predictive value for the presence of OAD. The diagnostic accuracy of CRP improved in combination with dyspnea and smoking history. CRP was associated with the severity of dyspnea as well as $\mathrm{FEV}_{1}$ and $\mathrm{FIV}_{1}$. Furthermore, CRP increased with the number of pack years. Altogether, these parameters might allow risk stratification of patients with OAD in primary care. Further studies need to be done to confirm these findings.

\section{Competing interests}

The author(s) declare that they have no competing interests.

\section{Authors' contributions}

AS designed the study, performed the statistical analysis and wrote the manuscript. GJD helped with the analysis and to write the manuscript. IM performed the spirometry and helped with the data analysis. LG performed the spirometry and documented the data. FJM helped to interpret the data and to write the manuscript. JS supervised the study and contributed in the writing of the manuscript.

\section{Acknowledgements}

We want to thank the participating GPs for allowing us to investigate the patients in their practices. We are grateful to Dr. Steffen Witte, Dept. of Medical Biometry, Heidelberg University for his statistical advice.

\section{References}

I. Murray CJ, Lopez AD: Alternative projections of mortality and disability by cause 1990-2020: Global Burden of Disease Study. Lancet 1997, 349: I498-1504.

2. van Schayck CP: Diagnosis of asthma and chronic obstructive pulmonary disease in general practice. $\mathrm{Br} J$ Gen Pract 1996, 46:193-197.

3. Holleman DR Jr, Simel DL, Goldberg JS: Diagnosis of obstructive airways disease from the clinical examination. J Gen Intern Med 1993, 8:63-68.

4. Straus SE, McAlister FA, Sackett DL, Deeks J]: Accuracy of history, wheezing, and forced expiratory time in the diagnosis of chronic obstructive pulmonary disease. J Gen Intern Med 2002, 17:684-688.

5. Grassi M, Rezzani C, Biino G, Marinoni A: Asthma-like symptoms assessment through ECRHS screening questionnaire scoring. J Clin Epidemiol 2003, 56:238-247.

6. Jackson $H$, Hubbard R: Detecting chronic obstructive pulmonary disease using peak flow rate: cross sectional survey. $B M]$ 2003, 327:653-654

7. Gan WQ, Man SF, Senthilselvan A, Sin DD: Association between chronic obstructive pulmonary disease and systemic inflam- 
mation: a systematic review and a meta-analysis. Thorax 2004 , 59:574-580.

8. Sin DD, Man SF: Why are patients with chronic obstructive pulmonary disease at increased risk of cardiovascular diseases? The potential role of systemic inflammation in chronic obstructive pulmonary disease. Circulation 2003, 107:15|4-1519.

9. Ferguson GT, Enright PL, Buist AS, Higgins MW: Office spirometry for lung health assessment in adults: $A$ consensus statement from the National Lung Health Education Program. Chest 2000, II7:II46-II6I.

10. O'Dowd LC, Fife D, Tenhave T, Panettieri RA Jr: Attitudes of physicians toward objective measures of airway function in asthma. Am J Med 2003, I | 4:391-396.

II. Taube C, Lehnigk B, Paasch K, Kirsten DK, Jorres RA, Magnussen H: Factor analysis of changes in dyspnea and lung function parameters after bronchodilation in chronic obstructive pulmonary disease. Am J Respir Crit Care Med 2000, 162:216-220.

12. Taube C, Kanniess F, Gronke L, Richter K, Mucke M, Paasch K, Eichler G, Jorres RA, Magnussen H: Reproducibility of forced inspiratory and expiratory volumes after bronchodilation in patients with COPD or asthma. Respir Med 2003, 97:568-577.

13. Burney PG, Luczynska C, Chinn S, Jarvis D: The European Community Respiratory Health Survey. Eur Respir J 1994, 7:954-960.

14. American Thoracic Society: Standardization of Spirometry. Am J Respir Crit Care Med 1995, I 52: I I07-I I 36.

15. National Institute of Health: Global Initiative for Asthma - Global Strategy for Asthma Management and Prevention 2004 [http://www.ginas thma.com].

16. Wilson EB: Probable inference, the law of succession, and statistical inference. J Am Stat Assoc 1927, 22:209-212.

17. Simel DL, Samsa GP, Matchar DB: Likelihood ratios with confidence: sample size estimation for diagnostic test studies. J Clin Epidemiol 1991, 44:763-770.

18. Bai J, Peat JK, Berry G, Marks GB, Woolcock AJ: Questionnaire items that predict asthma and other respiratory conditions in adults. Chest 1998, I | 4: | 343-1348.

19. Kilpelainen M, Terho EO, Helenius H, Koskenvuo M: Validation of a new questionnaire on asthma, allergic rhinitis, and conjunctivitis in young adults. Allergy 200I, 56:377-384.

20. Li JT, O'Connell EJ: Clinical evaluation of asthma. Ann Allergy Asthma Immunol 1996, 76: I-13.

21. Danesh J, Whincup P, Walker M, Lennon L, Thomson A, Appleby P, Gallimore JR, Pepys MB: Low grade inflammation and coronary heart disease: prospective study and updated meta-analyses. BMJ 2000, 32 I: 199-204.

22. Koh JM, Khang YH, Jung CH, Bae S, Kim DJ, Chung YE, Kim GS: Higher circulating hsCRP levels are associated with lower bone mineral density in healthy pre- and postmenopausal women: evidence for a link between systemic inflammation and osteoporosis. Osteoporos Int 2005, 16:|263-|27|.

23. Douglas KM, Taylor AJ, O'Malley PG: Relationship between depression and $\mathrm{C}$-reactive protein in a screening population. Psychosom Med 2004, 66:679-683.

24. Gan WQ, Man SF, Sin DD: The interactions between cigarette smoking and reduced lung function on systemic inflammation. Chest 2005, 127:558-564.

25. Huijnen B, van der HF, van Amelsvoort L, Wesseling G, Lansbergen $M$, Aarts $P$, Nicolson N, Knottnerus A: Dyspnea in elderly family practice patients. Occurrence, severity, quality of life and mortality over an 8-year period. Fam Pract 2006, 23:34-39.

26. Hopstaken RM, Muris JW, Knottnerus JA, Kester AD, Rinkens PE, Dinant GJ: Contributions of symptoms, signs, erythrocyte sedimentation rate, and $\mathrm{C}$-reactive protein to a diagnosis of pneumonia in acute lower respiratory tract infection. $\mathrm{Br} J \mathrm{Gen}$ Pract 2003, 53:358-364.

27. Hansen JG, Schmidt H, Rosborg J, Lund E: Predicting acute maxillary sinusitis in a general practice population. BMJ 1995, 31 I:233-236.

28. Bjerrum L, Gahrn-Hansen B, Munck AP: C-reactive protein measurement in general practice may lead to lower antibiotic prescribing for sinusitis. BrJ Gen Pract 2004, 54:659-662.

29. Knottnerus JA, van Weel C, Muris JW: Evaluation of diagnostic procedures. $B M J$ 2002, 324:477-480.
30. Hunter C], Brightling CE, Woltmann G, Wardlaw AJ, Pavord ID: A comparison of the validity of different diagnostic tests in adults with asthma. Chest 2002, 121:1051-1057.

31. Pekkanen J, Pearce N: Defining asthma in epidemiological studies. Eur Respir J 1999, 14:95 I-957.

32. Jenkins MA, Clarke JR, Carlin JB, Robertson CF, Hopper JL, Dalton MF, Holst DP, Choi K, Giles GG: Validation of questionnaire and bronchial hyperresponsiveness against respiratory physician assessment in the diagnosis of asthma. Int J Epidemiol 1996, 25:609-616.

33. Knottnerus JA: Medical decision making by general practitioners and specialists. Fam Pract 1991, 8:305-307.

\section{Pre-publication history}

The pre-publication history for this paper can be accessed here:

http://www.biomedcentral.com/1471-2296/7/28/prepub
Publish with Biomed Central and every scientist can read your work free of charge

"BioMed Central will be the most significant development for disseminating the results of biomedical research in our lifetime. "

Sir Paul Nurse, Cancer Research UK

Your research papers will be:

- available free of charge to the entire biomedical community

- peer reviewed and published immediately upon acceptance

- cited in PubMed and archived on PubMed Central

- yours - you keep the copyright
BioMedcentral 